\title{
Luigi Cavazza \\ Protagonista del progresso delle scienze agrarie
}

\author{
Angelo Caliandro* \\ Dipartimento di Scienze delle Produzioni Vegetali, Università di Bari \\ Via Amendola 165/a, 70126 Bari
}

\begin{abstract}
Autorità, illustrissimi Presidenti dell'AISSA e delle Società di Scienze Agrarie,

illustri Colleghi,

Signore e Signori,

ho accettato con immenso piacere e profonda commozione l'invito rivoltomi dal Presidente dell'AISSA, dott. Michele Stanca, a illustrare il contributo apportato all'evoluzione delle Scienze Agrarie dal prof. Luigi Cavazza, Emerito di Agronomia generale e Coltivazioni erbacee dell'Università di Bologna, durante la Sua lunga vita universitaria; il prof. Cavazza proprio quest'anno compie i sessant'anni di attività, essendosi laureato a Bari nell'anno accademico 1945/46. Il suo impegno di studioso dei problemi agronomici coincide con un periodo di profonda trasformazione dell'agricoltura italiana, rapidamente passata da un sistema arcaico ad uno moderno e innovativo grazie ai contributi della ricerca scientifica.
\end{abstract}

Ho avuto l'onore di conoscere il professor Cavazza da studente della Facoltà di Agraria dell'Università di Bari, quando frequentavo i corsi di agronomia generale, di coltivazioni erbacee e di metodologia sperimentale e ho avuto il piacere di collaborare al suo fianco fin dagli anni universitari. Questo ricordo mi riempie di commozione e orgoglio.

Le sue lezioni mi colpirono per il rigore scientifico e la trattazione multidisciplinare degli argomenti svolti, tanto che al momento della scelta della tesi di laurea, affascinato, senza indugio optai per un argomento agronomico.

Quando chiesi di poter svolgere la tesi di laurea in agronomia fui nuovamente colpito dalla metodologia usata per individuare con precisione l'argomento che coinvolgesse profondamente l'interesse del laureando: si avvaleva di un articolato e particolareggiato questionario che preliminarmente il laureando era tenuto a compilare. Allo stesso tempo mi colpì profondamente il rigore nell'applicazione degli schemi sperimentali per una significativa risposta ai risultati di campo, dal momento che ero abituato da giovanissimo nell'azienda paterna a una visione e opinione soggettiva nella gestione delle colture agrarie.

Il corso di metodologia statistica sperimentale, per studenti della Facoltà di Agraria e di altre Facoltà biologiche, veniva svolto a Bari per la prima volta per iniziativa del prof. Cavazza, convinto dell'utilizzo della statistica per una corretta interpretazione dei risultati della ricerca: era l'inizio della diffusione di metodologie sperimentali rigorose nell'ambito delle scienze agrarie e biologiche, di cui il prof. Cavazza continua a essere protagonista anche con la sua partecipazione, come docente, a corsi di metodologia sperimentale organizzati da istituzioni diverse.

Il prof. Luigi Cavazza ha iniziato la carriera accademica subito dopo aver conseguito la laurea in Scienze Agrarie presso l'Università di Bari sotto la guida del prof. E. Pantanelli, che, proveniente dalla Stazione agraria del Ministero dell'Agricoltura, nata a Bari nel 1920, per chiara fama, era stato nominato professore ordinario di Agronomia generale e coltivazioni erbacee e successivamente eletto Preside della

\footnotetext{
* Autore corrispondente: tel.: +39 080 5443031; fax: +39 080 5442976. Indirizzo e-mail: caliandro@agr.uniba.it.
} 
Facoltà di Agraria. Il prof. Pantanelli è stato uno studioso di fama internazionale e profondo conoscitore della realtà meridionale e seguendo il suo esempio, il prof. Cavazza ha continuato a dare prestigio alla scuola agronomica barese.

In un momento ancora difficile per i viaggi ed i contatti con istituzioni di altre nazioni, con grande spirito di sacrificio, per ampliare le sue conoscenze e assecondare la sua grande passione per lo studio e la ricerca scientifica nel campo agronomico, ha trascorso diversi periodi di soggiorno all'estero: presso l'Istituto di Fisiologia vegetale del Politecnico di Zurigo; presso il Dipartimento di Fisica, della Stazione sperimentale di Rothamsted (Inghilterra); presso l'Università di Logan, Utah, negli Stati Uniti, dove conseguì il Master of Science in Agronomia con tesi in Fisica del terreno con il prof. S.A. Taylor.

Dalla morte del prof. Pantanelli nel 1951 fino al trasferimento nella sede di Bologna ha contribuito al potenziamento dell'Istituto di Agronomia di Bari, dapprima come Aiuto e dal 1959 al 1969, vincitore di concorso della cattedra di Agronomia generale e Coltivazioni erbacee, come Direttore.

L'intensa attività di studio e di ricerca svolta dal prof. Cavazza con rigore scientifico, con ampiezza di interessi e con grande passione ed entusiasmo, trasmessa ai giovani ricercatori attraverso incontri, seminari e lezioni, è iniziata nel campo della fisiologia e analisi delle sementi ed è proseguita su problemi della fertilità del suolo e delle tecniche agronomiche.

Per molti anni ha dedicato la propria attività ad aspetti idrologici del sistema continuo suolopianta-atmosfera, occupandosi dei problemi dell'irrigazione, nonché degli aspetti fisici delle lavorazioni del terreno e della fertilità dei suoli.

La sua produzione scientifica, documentata da oltre 340 pubblicazioni, su riviste nazionali ed estere, tra cui uno studio sulla produzione delle foraggere nel mezzogiorno, un testo di fisica del terreno agrario e un testo, di recente pubblicazione, di applicazioni di fisica del terreno, in collaborazione con la prof.ssa Antonia Patruno, è caratterizzata, oltre che da interessanti e originali contributi alle problematiche affrontate, da innovative metodologie sperimentali e interpretazioni dei risultati ottenuti.

Ha operato sempre con il piacere di trasmettere agli altri il suo sapere e allo stesso tem- po con il desiderio di verificare nella pratica agronomica quotidiana le proprie convinzioni scientifiche. A questo riguardo merita di essere ricordato il felice incontro a Bari, all'inizio degli anni Cinquanta, del prof. Cavazza con il prof. Remigio Baldoni, uomini di differente esperienza e carattere, ma animati dalla stessa passione per la ricerca agronomica e dalla voglia di fondere insieme i principi teorici, che Cavazza domina, con la grande esperienza pratica di Baldoni. Questo periodo di intensa e proficua attività si protrasse per circa un quinquennio, fino a quando il prof. Baldoni non si trasferì a $\mathrm{Pa}-$ dova prima e a Bologna successivamente, dove Cavazza lo raggiunse alla fine degli anni Sessanta per riprendere l'intesa che si era stabilita a Bari.

Durante la permanenza a Bari la sua opera scientifica è documentata, oltre che dalle numerose pubblicazioni, dalla continua presenza a incontri di studio e dalla fattiva partecipazione alle realizzazioni di importanti opere, quali: la fitta rete di campi sperimentali durante il periodo di interventi della Cassa per il Mezzogiorno; l'azienda sperimentale "E. Pantanelli" della Facoltà di Agraria di Bari; l'Istituto Agronomico Mediterraneo di Bari, in Valenzano, attualmente una delle più importanti istituzioni internazionali operanti nel campo dell'irrigazione; l'ampliamento dell'Istituto di Agronomia generale e coltivazioni erbacee dell'Università di Bari, con criterio multidisciplinare di tipo dipartimentale (il prof. Cavazza è stato il primo in Italia a richiedere e ottenere lo sdoppiamento dell'insegnamento di agronomia generale e coltivazioni erbacee e a richiedere e ottenere il bando del concorso della cattedra di Miglioramento genetico delle piante agrarie); l'avvio del gruppo di studio dell'irrigazione del CNR, l'attuale GRUSI, costituito dai compianti professori Angiolo Crocioni (Torino), Raffaele Barbieri (Sassari e poi Napoli), Gianpiero Ballatore (Palermo) e dal prof. Luigi Cavazza (Bari); l'avvio della Rivista di Agronomia, organo ufficiale della Società Italiana di Agronomia, di cui è stato direttore responsabile per i primi otto anni di vita, dal 1967 al 1974.

Trasferitosi a Bologna ha continuato la sua intensa attività di studioso con immutato entusiasmo e piacere di operare offrendo alla comunità scientifica lodevoli contributi nel campo delle scienze agrarie; ha partecipato all'attività 
di ricerca sull'irrigazione del Consorzio di Bonifica di II grado per il Canale Emiliano-Romagnolo; è stato membro del Consiglio Superiore dell'Agricoltura e delle Foreste e membro del Comitato Tecnico dell'Autorità di bacino del Po; ha effettuato numerosi studi sull'utilizzazione irrigua dell'acqua in diverse aree della $\mathrm{Pu}$ glia e dell'Emilia-Romagna.

Sembrerà che mi ripeta, ma non mi stancherò mai di sottolineare che il prof. Cavazza, indipendentemente dalla sede nella quale si è trovato a operare, ha apportato il suo contributo all'evoluzione delle scienze agrarie, oltre che attraverso le numerose pubblicazioni, ciascuna delle quali merita un'attenta lettura per ben comprendere gli aspetti innovativi riportati, anche con l'attiva partecipazione a incontri di studio e convegni, pronto a intervenire per trasmettere preziosi suggerimenti di rigore scientifico, indirizzati particolarmente a giovani ricercatori.

La sua vasta preparazione scientifica, mai disgiunta dalla praticità, dalla semplicità e amabilità del carattere e dall'attenta osservazione della realtà agricola, pronto a recepire le opinioni dell'umile gente dei campi, e la sua grande capacità di trasmettere agli altri il suo sapere con passione ed entusiasmo sono state particolarmente manifeste durante il periodo di direzione della Rivista di Agronomia, con i prezioni suggerimenti scientifici agli autori nel revisionare i contributi ricevuti, e nell'ambito del GRUSI, di cui è stato animatore e coordinatore per circa un trentennio.

Nel GRUSI il prof. Cavazza è riuscito a creare un'atmosfera di calorosa, spontanea e multidisciplinare adesione di un folto gruppo di ricercatori che con regolarità s'incontra due volte all'anno per svolgere seminari, riguardanti l'irrigazione sotto tutti i suoi aspetti, e tenere cicli di conferenze di aggiornamento sulle varie discipline coinvolte nello studio dell'irrigazione, il tutto all'insegna del reciproco apprendimento; sempre nell'ambito del GRUSI, inoltre, si dimostrarono altamente formativi due corsi di aggiornamento in agronomia, svolti negli anni ac- cademici 1976-77 e 1977-78, autorizzati dal Ministero della Pubblica Istruzione.

Attualmente il prof. Cavazza continua a frequentare il GRUSI con assiduità e con la vivacità di sempre.

L'attività svolta dal prof. Cavazza e il contributo all'evoluzione delle scienze agrarie è stato apprezzato sia in Italia che all'estero, come dimostrano le numerose manifestazioni di riconoscimento, tra cui il Premio Justus von Liebig della Fondazione FVS, assegnato dall'Università di Kiel nel 1973; il Premio della Fondazione Marchi, assegnato dall'Accademia dei Georgofili nel 1992; il Sigillo d'oro dell'Università degli studi di Foggia, offerto nel 2005.

Il prof. Cavazza è stato presidente dell'Accademia nazionale dell'Agricoltura, dell'UNASA (Unione Nazionale delle Accademie italiane per l'agricoltura, la sicurezza alimentare e la tutela ambientale) e della Società Italiana di Agronomia; è accademico emerito dei Georgofili, accademico corrispondente dell'Accademia di Agricoltura di Francia, socio onorario dell'Accademia Gioenia, socio emerito dell'Associazione Idrotecnica Italiana; è Cavaliere del Ministero dell'Agricoltura di Francia.

Interpretando il pensiero dei presenti all'incontro odierno, con spirito di stima e di profonda riconoscenza e gratitudine, ringrazio, quindi, l'illustre professore Luigi Cavazza, maestro di scienza e di vita, per la sua opera a favore del progresso delle scienze agrarie ed esprimo la mia gratitudine al Presidente dell'AISSA per aver voluto affidarmi il prestigioso incarico di illustrare la figura dello scienziato.

Certamente non sono stato completo nella mia esposizione, un pò smarrito nella copiosa raccolta di testimonianze di sessant'anni di intensa vita da studioso, pur tuttavia sono lieto di augurare al prof. Cavazza una lunga vita tra noi e negli affetti più intimi della Sua famiglia, e prima di concludere questo breve intervento vorrei che il prof. Cavazza ci facesse una promessa, quale ulteriore dono del Suo sapere: portare a compimento il trattato di agronomia da tempo avviato. 